\title{
Development of solar shading system for preventing driving accidents using transparent display
}

\author{
*Shintaro Matsuo, Siichi Serikawa, Shiyuan Yang \\ Department of Electrical and Electronic Engineering, Kyushu Institute of Technology, Kitakyushu, Japan \\ *Corresponding Author: matsuo.shintaro931@mail.kyutech.jp
}

\begin{abstract}
Many traffic accidents occur every day. There are reasons causing them. One of the causes is sunlight glare. This study proposes a new method to solve this. We develop a system that automatically blocks sunlight using a LCD panel and camera. The LCD panel used in this study can be controlled to a portion that transmits light and a portion that blocks light. This study uses two cameras. A camera takes the front of a car and the other takes a driver's face. The positions of the sun and the position of the driver's eyes are calculated from the images taken by each camera. The light shielding area on a LCD is determined from the calculated positions. As a result, it is possible to block only the sunlight portion on the LCD without blocking the wide view.
\end{abstract}

\section{Introduction}

With the widespread use of cars, people's movement has become very easy and convenient. However, a possibility to have traffic accidents is increasing. There are still many traffic accidents. In a traffic accident, some people may become handicapped or lose their life. Thus, the research to prevent traffic accidents is very important. In recent years, sensing technology to prevent traffic accidents has been very developed and some of them are in practical use.

Traffic accidents are caused by various factors. The factors are divided into two. (1) One is caused by a driver, and (2) the other is caused by an environment. Factor (1) includes unconfirmed safety, driving aside, and distraction. Factors (2) include poor visibility in the rain and poor visibility at intersections. The dazzling sunlight is also one of the factors .

There are several methods to reduce the glare of sunlight while driving. The most common method is to attach a sun visor. This is easy to attach, but greatly obstructs the driver's view. Other methods include attaching a translucent film or transmissive liquid crystal. The film does not obstruct the driver's view very much, but it is less effective for preventing strong sunlight. The method using a transmissive liquid crystal shields the all area of liquid crystal when sunlight is detected, it is resulting in poor visibility like a sun visor.

In this way, the conventional methods have advantages and disadvantages, respectively. Therefore, it is necessary to develop a new system that does not block a driver's view in spite that the sun light is blocked during driving. In this study, the position of the sun light and the position of the eyes of a driver are detected using two cameras. Using the results, we will develop a system that controls a transmissive liquid display, blocks only the sun, and transmits the other areas. Thereby, the block of sun light and the good visibility can be realized at the same time.

\section{Method to block out only sun light}

\subsection{Method for detecting sun light position by image processing}

In general, the sun light is the brightest light in the natural environment. In this study, the brightest area in the image is regarded as the sun light area and the area is blocked. Images captured by a camera are represented by the brightness of each RGB pixel. Therefore, it does not represent the luminance value. Therefore, the brightness of each RGB pixel is converted to $\mathrm{YCrCB}$ space using following equations (1)-(3). Equation (1) represents the luminance value. Figure 1 shows how sun light is detected and blocked. Figure 1(a) represents the original image, and 
Fig.1(b) represents the detected image. As understood from Fig. 1(b), we can see that the position of the sun is correctly detected and it is pasted as black.

$$
\begin{gathered}
Y=0.229 R+0.587 G+0.114 B \\
C_{r}=-0.169 R-0.331 G+0.500 B \\
C_{b}=0.500 R-0.419 G-0.081 B
\end{gathered}
$$

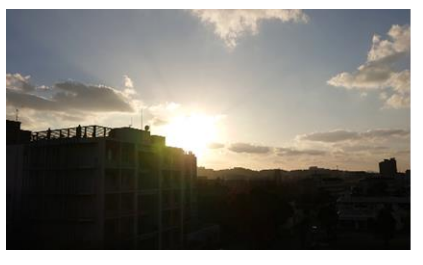

(a) Original image

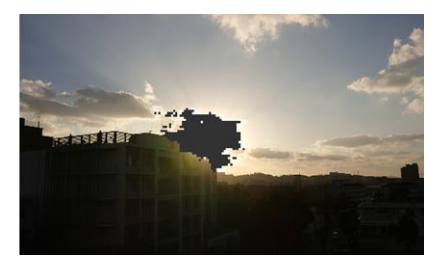

(b) Detected image
Figure 1 Detection of sun light

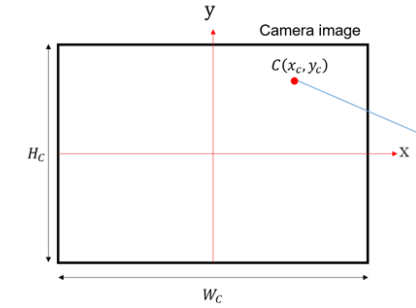

(a) Camera image (b) LCD image

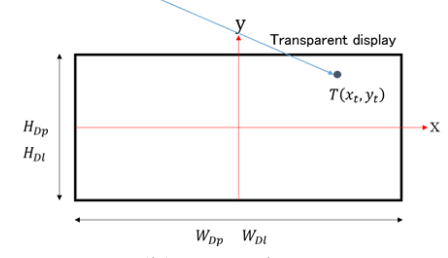

Figure 2 Camera image and the LCD image.

\subsection{Detection of light blocking position}

As shown in Fig. 2, the image viewed from a camera is different from the image viewed from the transmissive liquid crystal display (LCD) through the human eyes. Therefore, it is necessary to make the position of the image from the camera correspond to that of LCD. Here, the center position of the LCD is set to be the origin point $(0$, $0)$.

Figure 3 shows the positions of eyes, camera, LCD and the sun. Here the camera is considered to be put horizontally. Let the angle by the sun be $\theta$ when the horizontal direction is 0 degrees as shown in Fig. 3. This angle $\theta$ can be easily obtained by image processing. Since the distance to the sun is far enough, the angle of the sun obtained from the camera and that from from the driver's eye can be considered to be the same. From the distance $\mathrm{L}$ between eye and LCD and the acquired angle $\theta$, the position of shield on the LCD can be calculated. We define the variables as follows:

Camera angle of view, $\left(\theta \_c, \varphi \_c\right)$ [degrees]. Number of camera pixels, (W_c, H_c) [pixels]. Image location, (x_c, y_c) [pixels]. Distance from eye to display, L [mm]. LCD pixel count, (W_Dp, H_Dp) [pixel]. LCD size, (W_Dl, H_Dl) [mm].

Then, the position ( $\mathrm{x}_{-} \mathrm{t}, \mathrm{y} \_\mathrm{t}$ ) [pixel] of the sun to be displayed on the LCD is as follows.

\begin{tabular}{|c|c|}
\hline$x_{t}$ & $=L \tan \left(\frac{\theta_{c} x_{c}}{W_{c} / 2}\right) \frac{W_{D p}}{W_{D l}}$ \\
\hline$y_{t}$ & $=L \tan \left(\frac{\varphi_{c} y_{c}}{H_{c} / 2}\right) \frac{H_{D p}}{H_{D l}}$
\end{tabular}

\subsection{Correction of light blocking area by binocular parallax}

The distance between human right and left eyes is about $6 \mathrm{~cm}$. As a result, the images in the retina of the right eye and left eye are slightly different. For this reason, the position of sun light with the right eye and the position of sun light with the left eye are different. Therefore, it is

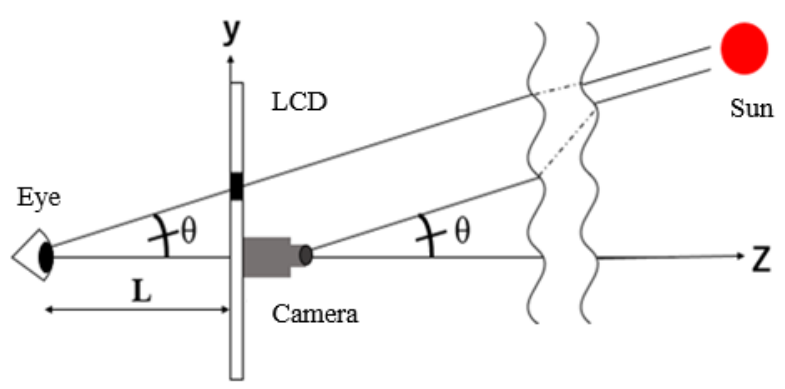

Figure 3 Positions of eyes, camera, LCD and the sun necessary to obtain two light shielding areas corresponding to each the eyes. In this case, the position of pupils are detected by the Web camera, and two light shielding area corresponding to each position of the right eye and the left eye is obtained.

\section{Proposed system}


Figure 4 shows a schematic diagram of the system proposed in this study. It consists of Web cameras W1 and $\mathrm{W} 2$ and an LCD, and PC. The W1, W2, and LCD are connected to a PC. Web camera W1 captures the front of the car in real time and detects the position of the sun. The driver is captured by the Web camera W2, and the positions of eyes are detected by image processing. Using these detection results and Equation (4) and (5), we calculated the sun light blocking area on the LCD.

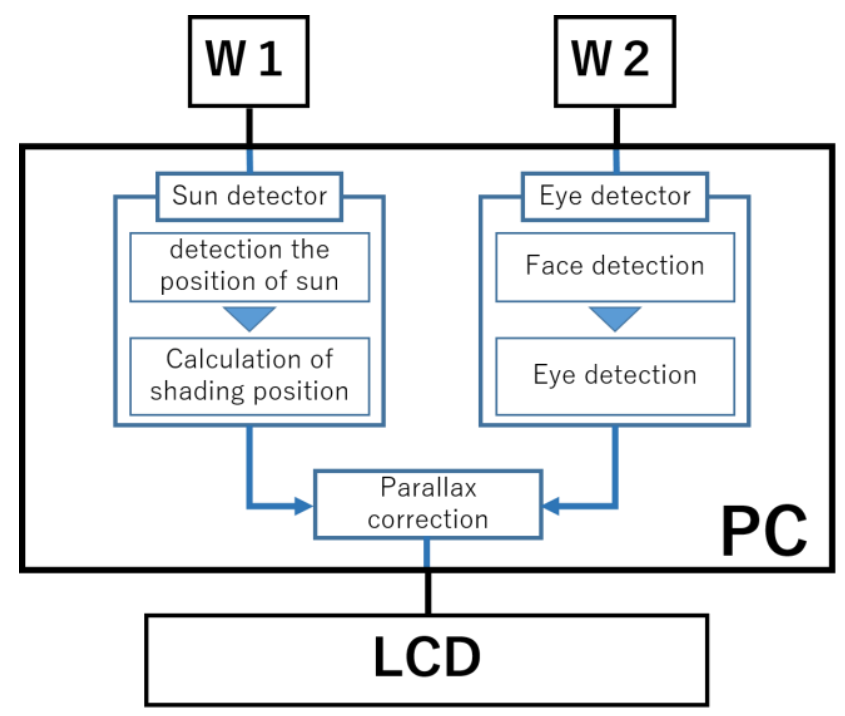

Figure 4 Proposed solar shading system.

\section{Experimants}

\subsection{Verification of sun light blocking in monocular}

In this experiment, we verified the blocking of sun light in case of a single eye. Here, since it is necessary to check at various light positions, we experiment using LED light (total luminous flux $1800 \mathrm{~lm}$ ) instead of actual sun light. First, a LED light is set to be the center of the image captured by the Web camera W1. Next, let move the single eye and adjust the viewpoint so that the light from the LED is centered on the LCD. In addition, the sun light area on the LCD is blocked. This is the initial position. Here, the distance between the eye and the LCD is fixed at $35 \mathrm{~cm}$.

In this condition, it was verified whether the light source area was correctly blocked by moving the LED light. The results are shown in Fig. 5. As understood from Fig. 5, we can see that the position of the light source is correctly blocked even if the LED light is moved.

\subsection{Verification of sun light blocking with both eyes}
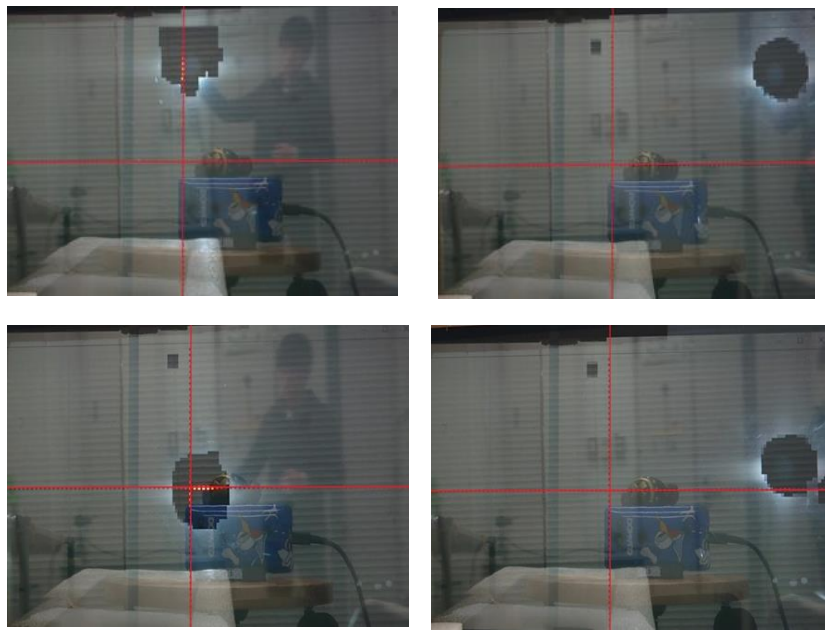

In Section 4.1, in the case of single eye, it was found that the position of the light source can be specified and the Figure 5 Result blocked of sun light position in monocular

position of the light source on the LCD can be blocked. When using both eyes, the image projected on retina in right eye is slightly different from that of left eye. Therefore, it is necessary to calculate the both positions corresponding to the left and right eyes and to block the two areas on the LCD. That is verified here.

First, a LED light is set to be the center of the image captured by the Web camera W1. Next, while maintaining the distance from the eyes to LCD at $30 \mathrm{~cm}$, the face moves vertically and horizontally. Camera W2 always detects two eye's positions on a face. By detecting the eyes position and light source position, the blocking position on the LCD is automatically calculated. We checked whether the positions of the light source were correctly blocked while moving the face. A face is actually moved in 4 directions. The moved position is shown in Fig. 6. Figure 7 shows the result of blocking the light source in 4 directions.

As understood from the figure, the position of the light source moves according to the movement of the face, and the light source on the LCD is correctly blocked.

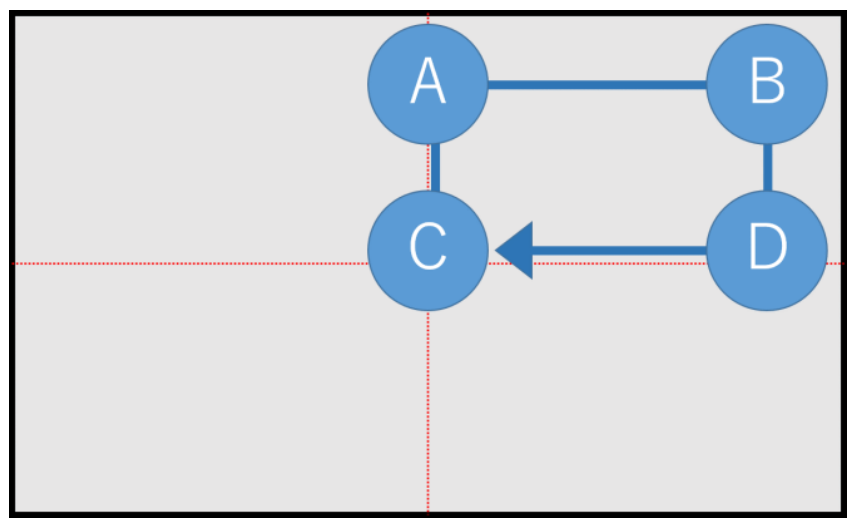

Figure 6 Movement positions of both eyes 

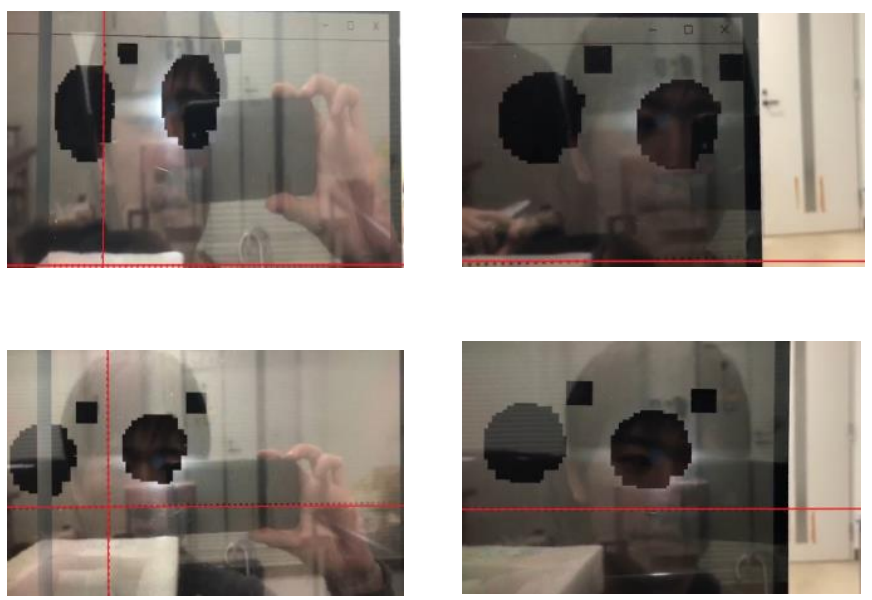

Figure 7 Result of blocking the light source in 4 directions.

\section{Investigation}

From the experimental results by the monocular in 4.1 , it was found that the light source was correctly blocked even when the light source was moved in various directions. However, a part that were not light sources were sometimes blocked. In this study, the light source was determined by only the luminance value. In the future, it will be necessary to extract the light source region more correctly.

In some cases, the blocking range was slightly smaller than that of sun light. For the temporal improvement, the slightly larger area was blocked than that of the light source. The size should be optimized in the future.

From the experimental results in 4.2 , it was found that even if the face position was moved, two blocking areas corresponding to the left and right eye positions were made correctly. However, there was a time delay in image processing compared to actual facial movements. In particular, it was found that there was a delay caused by the transfer of image from the camera. This should be improved.

\section{Conclusion}

In this study, in order to reduce the glare of sun light while driving, we developed a system that uses an LCD to block out only the sun light region. The system was actually developed and verified whether it was useful or not.

At First, sun light position was detected in real time from images taken with a Web camera. Next, it was shown that only glare field can be blocked by controlling the transmissive liquid crystal display (LCD). In addition, the eye position was always traced using another camera. If we move the face position, the blocking area also moves dynamically. As a result, sun light glare can be automatically blocked and not to block the other area while driving.

In this study, there were rare cases where the position of sun light could not be extracted correctly. There was also a slight time delay from the time of camera capture to display on LCD. These should be improved in the future.

\section{References}

(1) Kenji Hagita and Kenji Mori. 2011. "An Analysis of Traffic Accident Influenced by Glare of the Sun". J.Society of Civil Engineers.Jpn.67,5(in Japanese) 